\title{
Cauda equina syndrome in dogs - a review
}

\author{
Igor Šulla1, Vladimír Balik², Slavomír Horňák ${ }^{3}$, Valent Ledecký3 \\ ${ }^{1}$ University of Veterinary Medicine and Pharmacy, Department of Anatomy,Histology and Physiology, \\ Košice, Slovakia \\ ${ }^{2}$ Palacky University, Institute of Molecular and Translational Medicine, Olomouc, Czech Republic, \\ ${ }^{3}$ University of Veterinary Medicine and Pharmacy, Small Animals Clinic, Košice, Slovakia
}

Received February 17, 2018

Accepted December 7, 2018

\begin{abstract}
A lesion of sacrococcygeal spinal nerve roots forming a structure that resembles a horse's tail results in the development of clinical entity identified as the cauda equina syndrome (CES). The disease can evolve slowly and symptomatology can be incomplete, but the fully developed CES is characterized by pain and altered sensation in the pelvic extremities, tail, perianogenital region, paresis or plegia of hind limbs, incontinence and impotence. Major causes of CES in dogs are degenerative changes of the lumbosacral vertebral column, haematoma, inflammation, neoplasm or trauma. The diagnosis is based on history, clinical presentation, neurological symptomatology, spinal röntgenography, computed tomography, and magnetic resonance imaging. In animals experiencing initial episodes of CES, conservative therapy can be attempted. But the only rational treatment of patients with severe neurological deficit is surgical decompression of the neural structures. The outcome depends on the underlying aetiology and the degree of sensory, motor and autonomic dysfunction. Canine and porcine experimental models mimicking the CES showed the involvement of intrinsic spinal cord structures. This points out the need for an early diagnosis followed by aggressive management before irreversible neuronal lesions develop. The search strategy involved the PubMed, Medline, Embase and ISI Web of Science from January 2000 to August 2017 using the terms 'cauda equina syndrome' and 'lumbosacral stenosis' in the English language literature; also references from selected papers were scanned and relevant articles included.
\end{abstract}

Dog, cauda equina syndrome, diagnostics, therapy, prognosis

The cauda equina syndrome (CES), also reported as cauda equina compression or degenerative lumbosacral stenosis (DLS) in the literature, is a neurological disorder that usually occurs in older, large or medium-sized working dogs with a male predisposition (Linn et al 2003; Worth et al. 2009). The CES develops as a result of simultaneous lesion of multiple lumbosacral (LS) nerve roots which look like the tail of a horse, i.e. cauda equina (CE) in Latin (Fletcher 2013). The clinical symptomatology varies according to the aetiology, degree of compression and individual differences. The early signs of CES can be inconspicuous or confusing until serious neurological deficit develops (Dewey 2008). The aim of this paper was to provide veterinarians with an overview of current knowledge related to CES in dogs.

\section{Essential morphological features}

The spinal cord (SC) enclosed within the vertebral canal is a direct continuation of the brain stem. The SC in dogs has 36 segments in total (8 cervical, 13 thoracic, 7 lumbar, 3 sacral, 5 caudal); extending from the $1^{\text {st }}$ cervical nerves to the caudal part of the elongated cone and consisting of spinal cord segments S2, S3, Cd1-Cd5 (Fletcher 2013). About $1 \mathrm{~cm}$ caudal to the last segment, the SC is reduced to a strand of glial and ependymal cells called the terminal filament. The medullary cone in giant dogs is located at the L6/L7 intervertebral space, in medium dogs at the L7/S1 intervertebral space, in small ones at the

Address for correspondence:

MVDr. Igor Šulla, $\mathrm{PhD}$

University of Veterinary Medicine and Pharmacy,

Department of Anatomy, Histology and Physiology

Komenského 73, 04181 Košice, Slovakia

Phone: +903 764161

E-mail: igor.sulla@uvlf.sk

http://actavet.vfu.cz/ 
level of S1 vertebra (Palmer and Chambers 1991; Fletcher 2013). The sacral and caudal spinal roots continue within the vertebral canal caudally and exit through the respective intervertebral foramina. At their level, the spinal roots join and become a spinal nerve. At the terminal part of every dorsal nerve root is an aggregation of pseudounipolar neurons named the spinal ganglion. Outside the vertebral canal, the L6, L7 and S1 spinal nerves converge to become a sciatic nerve; the S1-S3 spinal nerves form pelvic and pudendal nerves; the Cd1-Cd5 form caudal nerves (Palmer and Chambers 1991; Fletcher 2013). The spinal cord and CE inside the vertebral canal are enveloped by meninges (Plate I, Fig. $1)$.

The vascular supply of CE is derived from arteries which run along the ventral and dorsal roots. Each spinal nerve is usually accompanied by one small artery. They are so called "end arteries" with no effective anastomotic connections to the vasocorona (the surface vessels) of the spinal cord. The compression of radicular artery at any point may result in ischaemia of the nerve root. The blood flow in CE continues via their arborized venules and radicular veins to the right and left vertebral sinuses (Fletcher 2013; Mazensky et al. 2017).

The dorsal portion of the vertebral canal in the LS region is composed of the laminae of the L7 vertebra and the sacrum, the interarcuate ligament (yellow ligament) spans the space between the arches of L7 and S1 vertebra (Palmer and Chambers 1991; Scharf et al. 2004). The ventral portion of the canal is composed of the body of the L7 vertebra, the dorsal annulus fibrosus of the L7/S1 intervertebral disc, and the body of the sacral bone. The arches of the L7 and S1 vertebrae are linked by two joints at articular facets. The fibrocartilaginous joint between the L7 vertebra and sacral bone is subjected to the greatest stress during locomotion (Brisson 2010; Bergknut et al. 2013). It is stabilized by two longitudinal ligaments (ventral and dorsal), the L7-S1 yellow ligament, L7-S1 interspinal ligament, and paraspinal muscles. The canal in the L7 vertebra and sacral bone is dorsoventrally flattened, and so the nerve roots passing over the L7-S1 disc are more endangered by compression. The cranial part of the L7/S1 foramen is formed by the caudal notch of the L 7 vertebra, its caudal part by the cranial notch of the sacrum, the ventral part by the intervertebral disc, and the dorsal part by the articular processes and joint capsules (Rossi et al. 2004; Worth et al. 2009).

\section{Pathophysiology of the cauda equina syndrome}

The CES is a multifactorial pathological condition caused by entrapment of several, eventually of all CE spinal roots, which results in changes in the intraradicular blood flow and in nerve fibre dysfunction (Rydevik 1993; Otani et al. 2001; Lorenz et al. 2011). The mechanical constriction may also lead to different neural tissue reactions, e. g. oedema formation, demyelination, and fibrosis (Kobayashi et al. 2004). The porcine CES model revealed that venules in the CE begin to occlude at the pressure of $5 \mathrm{~mm} \mathrm{Hg}$. The blood flow in arterioles stopped when the compressing force surpassed the mean arterial pressure (Olmaker et al. 1992). Canine experimental studies showed that $10 \mathrm{~mm} \mathrm{Hg}$ compression affected the nerve conduction velocity (Yoshizawa et al. 1989; Otani et al. 2001). This explains the characteristic motor disturbance known as intermittent neurogenic claudication - the typical symptoms that appear after lumbar spine extension and that are relieved after a change of position (De Risio et al. 2000). It suggests that extension of the L7-S1 junction along with the propulsion forces acting on L7-S1 disc may cause a critical narrowing of the spinal canal (Worth et al. 2009; Meij and Bergknut 2010). It is generally accepted that pain in the LS region results from irritation of vertebral bodies, ligaments, the facet articular cartilages and joint capsules; radicular pain results from direct irritation of dorsal roots (Palmer and Chambers 1991; Meij and Bergknut 2010). It projects to their dermatomes and is often associated with paraesthesias (Lorenz 
et al. 2011). Additional pathomechanisms of radicular pain can be nerve root irritation induced by proteoglycans released from the intervertebral discs, an increased level of lactic acid, and a decreased pH (Rydevik 1993; Orendacova et al. 2001). Neuropathologic changes in S1-Cd5 dorsal root fibres were observed, accompanied by the degeneration of small neurons located in Rexed lamina VII, and by the retrograde degeneration of motoneurons in the ventral horn (Rexed lamina IX) of the S1-S3 spinal cord segments following 9-day constrictions of CE in a canine experimental model (Marsala et al. 1995).

\section{Aetiology of the cauda equina syndrome}

Important pathologic processes creating CE compression in dogs are (DLS), neoplasms, haemorrhage, infection, and trauma. The most common cause of CES in dogs are changes in the L7-S1 joint and the surrounding structures. They are responsible for the transmission of propulsion deriving from the pelvic extremities (Linn et al. 2003; Worth et al. 2009; Bergknut et al. 2013). The forces acting on this segment of the vertebral column are mostly those of flexion and extension. They are strong, and so the lumbo-sacral articulation and L7-S1 intervertebral disc predispose to degeneration (Hansen type II disc disease). The DLS or LS vertebral canal stenosis is an acquired degenerative disease causing reduction in the diameter of the lumbosacral spinal canal and its foramina (Palmer and Chambers 1991; Worth et al. 2009). As a consequence, the nerve structure compression appears, accompanied with an inflammatory reaction in the $\mathrm{CE}$ and adjacent structures (Tan et al. 2013).

The dogs with lumbosacral transitional vertebrae (LTV), especially those with asymmetrical LTV, are affected more often and at younger age with DLS and CES (Flückiger et al. 2006). The altered mobility and distribution of propulsion forces in lumbosacral joints cause premature degeneration of the intervertebral discs, malarticulation/ malformation of the lumbosacral junction combined with dysplasia of the articular processes of L7/L8 vertebrae leading to CES, sometimes with a unilateral preponderance of symptoms (Lappalainen et al. 2012). The highest prevalence of LTV was observed in German Shepherds (Scharf et al. 2004; Lappalainen et al. 2012; Fialová et al. 2014).

Many different types of benign or malignant neoplasms (arising from nerve roots, meninges, lumbar vertebrae, sacral bone, or metastatic) may affect the CE by compression or infiltration (Bagley and Gokaslan 2004). The most common primary malignant tumours occurring in this region are osteo-, chondro- or fibrosarcomas, less frequent are plasmocytomas. The benign neoplasms are meningiomas, Schwannomas, neurofibromas, or ependymomas (Bagley and Gokaslan 2004; Pang and Argyle 2016). A typical feature of neoplastic CE compression is an inconspicuous onset and slow progression of symptoms, until the critical point is reached. Then, the situation suddenly impairs (Dewey 2008; Lorenz et al. 2011). The proximity of the lumbosacral junction to the sublumbar lymphatic vessels and nodes, responsible for the drainage of the perineal area, prostate, uterus, rectum, urinary bladder and pelvic limbs, make this area a preferred location for metastases. The symptomatology mimicking CES can also appear in the case of neoplasm affecting the nerves formed from the L5-Cd5 spinal nerve roots, e. g. the sciatic nerve (Trebacz and Galaty 2016).

An epidural haematoma (EDH) is a rare spinal pathology (Orendacova et al. 2001; Kebaish and Awad 2004; Minish et al. 2015). Its clinical presentation depends on its location and the degree of SC/CE compression. It manifests itself by a combination of pain and neurological deficit. The spinal EDH is most frequently caused by spontaneous venous bleeding, but vertebral fractures, lumbar puncture, vascular anomalies, and spinal tumours can be aetiological factors, too (Kebaish and Awad 2004).

The most common infection of the lumbosacral junction is discospondylitis (Velissaris et al. 2009; Rutges et al. 2016). Sometimes multiple discs are affected. The aetiology 
is bacterial or fungal; microorganisms gain access to the disk by haematogenous way, eventually they are inoculated directly in cases of penetrating injuries or therapeutic interventions (Nota et al. 2015; Rutges et al. 2016). Discospondylitis is extremely painful. Dogs afflicted with this disease suffer from a progressive paraparesis and ataxia. They have fever and are seriously ill (Rosc-Bereza et al. 2013). The diagnosis is facilitated by MRI and bacteriological tests (Lorenz et al. 2011; Rosc-Bereza et al. 2013). If spinal epidural abscess (SEA) or spinal subdural empyema (SSE) develops, the situation constitutes an emergency (Velis saris et al. 2009).

Traumatic CES, especially caused by traffic accidents, are a common problem in dogs. It should be considered in cases with an acute form of presentation (Orendacova et al. 2001; Thongtrangan et al. 2004). The patient may suffer significant damage to several body systems that pose more immediate threats to its life than an injury of CE. Therefore, when evaluating an animal with a neurologic deficit due to suspect trauma it is necessary to assess the overall stability of the organism (Webb et al. 2010). The management is modified according to the specific clinical situation.

\section{Clinical symptomatology}

The affected animals are usually large or medium sized, older, male dogs, with German Shepherds prevailing. Other affected breeds include Great Danes, Airedale Terriers, Irish Setters, English Springer Spaniels, Boxers, and Labrador and Golden Retrievers (Priester 1976; Worth et al. 2009; Brisson 2010). The CES is presented in dogs by a combination of clinical signs caused by damage to L7-Cd5 nerve roots (Dewey 2008; Worth et al. 2009). The most common symptom is lumbar pain (Worth et al. 2009; Lorenz et al. 2011). It may be caused by a nerve root entrapment, damage to the annulus fibrosus, degenerative changes of the articular processes and joints, or direct irritation to the dura mater. A pressure exerted over the lumbosacral area, hyperextension of the lumbar spine, and hip joint extension evoke symptoms of pain and discomfort (De Risio et al. 2000; Mayhew et al. 2002; De Decker et al. 2014). Occasionally, dogs may keep a pelvic limb off the ground (root signature), paraparesis may also develop. Sometimes dogs assume a crouched stance with overflexion of the hip, stifle and hock joints. They are characterized by a shortened and choppy stride, often yelp and hold up the limb. The paresis may result in a dog standing on top of the toes (knuckling) and subsequently ambulating on the dorsum of the paw (Palmer and Chambers 1991; Lorenz et al. 2011). Pelvic limb lameness is usually pronounced when the dog goes upwards or rises from a prone position. Pelvic limb lameness and weakness worsen with exercise. Animals may be reluctant to raise or wag their tails. Exercises and jumping are often avoided because they result in pain (De Risio et al. 2000). The impairment of motor functions also results in weakness or paralysis of the tail, manifested by inability to wag the tail or raise it during urination or defecation. The loss of sensation (analgesia) or abnormal sensation (dysesthesia or hypesthesia) and pain (hyperpathia) can result in licking, chewing or self-mutilating the back of the dog's thighs, lateral regions of the feet and toes, perineum, tail, genitalia (Mayhew et al. 2002). The most advanced cases can evolve into full paralysis. Abnormalities of urination, defecation and sexual functions are commonly associated with lesions of sacral nerve roots, too (McMurray et al. 2006; Lorenz et al. 2011). Urinary incontinence in dogs is highly variable. It ranges from occasional overflow of urine during sleep to constant dribbling. The urinary bladder is flaccid and distended as a result of a poor detrusor reflex, while the sphincter tone is still maintained, since innervation by the hypogastric nerve remains intact (Fowler 1999; McMurray et al. 2006). Abnormalities of defecation include inability to maintain a posture or to change a position, straining in defecation, and faecal incontinence. The anal sphincter can be atonic and the perineal reflex weak or absent (Lorenz et al. 2011). Dogs afflicted with CES manifest two types of sexual problems. 
Pain in the LS region hinders copulatory activities, i.e. mounts and intromissions in males, lordosis in females (McMurray et al. 2006). Severe CE lesions disrupt reflex-mediated increase in the genital blood flow (required for engorgement of erectile tissues in males and vasocongestion of clitoris, vaginal wall, and lubrication of the vaginal surface in females). Even if the sympathetically mediated arousal is intact, males have problems to maintain erection and suffer of retrograde ejaculation due to incompetence of the internal urethral sphincter (McMurray et al. 2006). As a result, male reproductive function is significantly affected. On the contrary, female dogs with CES are able to become pregnant, undergo normal pregnancy, and delivery (McMurray et al. 2006).

\section{Diagnostics}

The diagnosis of CES in dogs is based upon a careful analysis of the dog's history obtained from the owner, correct interpretation of physical findings, results of ancillary testing and imaging methods (Meij and Bergknut 2010; De Decker et al. 2014). Large breeds, medium to older age, and male sex are factors that increase the probability of occurrence of a degenerative lumbosacral stenosis in affected dogs. Back pain and radiculopathy accompanied with heterogenous neurological symptomatology suggest the presence of epidural or subdural haemorrhage; hyperpyrexia, excessive pain, sudden rise of serious neurologic deficit, and prostration are characteristic for spinal infections; slow progression is typical of neoplastic expansive lesions; an acute CES is usually caused by trauma (Linn et al. 2003; Dewey 2008; Webb et al. 2010; De Decker et al. 2014; Nota et al. 2015). A microbiological test and histopathologic examination of tissue samples are necessary for the indication of appropriate treatment strategies in intraspinal inflammations or tumours (Pang and Argyle 2016; Rutges et al. 2016).

\section{Neurological examination}

The animal is usually presented with a history of lumbosacral pain. The gait may be choppy with a plantigrade stance of pelvic limbs. A postural reaction deficit may be observed with hopping or hemiwalking. Proprioceptive positioning may be delayed, the withdrawal reflex reduced. The cranial tibial and gastrocnemius reflexes may be reduced or absent. In some animals, the patellar reflex appears brisk (patellar pseudohyperreflexia) due to loss of resistance from the flexors innervated by the sciatic nerve (Dewey 2008; Meij and Bergknut 2010). With the passive range of motion of the palvic limb, it is possible to observe hypotonia of a quality of the paresis or paralysis. Similarly, decreased tail tone may be apparent (Scharf et al. 2004; Lorenz et al. 2011). On palpation, the examiner can detect atrophy of the semimembranosus, semitendinosus, gastrocnemius, and cranial tibial muscles. The perineal reflex may be reduced or absent. Rectal examination may reveal a dilated sphincter. The bladder may be enlarged, but urine is easily expressed manually. Alternatively, one may notice constant dribbling of urine; the perineum wet with urine is an important symptom of incontinence in female dogs (Palmer and Chambers 1991; Suwankong et al. 2008). The urinalysis may reveal bacteriuria secondary to urethral sphincter incompetence (Fowler 1999).

Electrodiagnostic studies, especially electromyography and somatosensory evoked potentials, routinely employed in human neurology, are gaining growing popularity in veterinary medicine, as well (Shahmohammadi et al. 2016). Electromyography which is able to identify the impaired neural conduction to muscles of pelvic extremities, perineum and tail can be very helpful in differential diagnosis between CES and orthopeadic diseases mimicking this clinical entity. Somatosensory evoked potentials elicited by stimulation of peripheral branches of $\mathrm{CE}$ nerve roots (e.g. the tibial nerve) may be used in monitoring the recovery of neural functions and in the assessment of the effect of employed therapy (Szilasiová 2011). 


\section{Lumbar cerebrospinal fluid analysis}

In dogs, cerebrospinal fluid (CSF) for analysis is collected from the cerebellomedullary cistern or the lumbar subarachnoid space. The important things to follow in CSF are the total nuclear cell count, differential cell count, total protein concentration, bacteriological and cytological examinations; however, changes are not specific. The CSF analysis in dogs with spinal pathology is useful only when the results are correlated with the history, clinical findings and imaging studies (Dewey 2008; Lorenz et al. 2011). To establish the diagnosis by this method is possible only in situations where microorganisms or neoplastic cells are identified in CSF samples (Musteata et al. 2013; Hugo et al. 2014).

\section{Imaging diagnostic techniques}

Diagnosis of different abnormalities of the lumbosacral vertebral canal relies heavily on the imaging procedures (Mayhew et al. 2002; Brisson 2010). The most frequently used techniques are plain röntgenography (Plate I, Fig. 2), dynamic X-rays (i. e. laterolateral (LL) radiograms of vertebral column in flexion and extension), CT, and MRI. Plain X-ray remains not only a useful screening method, but dynamic x-ray images can show the instability of the L-S junction (Hylands 2007; Da Costa and Samii 2010). On the other hand, plain X-rays do not supply information on the soft tissues (Hylands 2007). Invasive techniques (myelography, epidurography, discography, spinal venography), used in the pre-CT era, are considered obsolete at present (Rossi et al. 2004).

The CT supplies images without superimposition of the surrounding tissues and allows CT reconstructions in different planes (Plate II, Fig. 3). The resolution of CT is excellent for hard tissues (the density of bones is 700-1000 HU) and very good for fresh blood (40-90 HU), but soft tissues (ligaments, nerve roots) are not visualized with the same level of detail (Lee et al. 2013). On the contrary, the presentation of fresh blood or disorders of bony structures by MRI is less reliable. That is why CT is an excellent method in diagnostics of vertebral fractures/luxations (especially when routine examination is completed with the utilization of a 3D-reconstruction technique) and epidural or subdural haematomas. In CES caused by intraspinal bleeding, CT shows a distinct, hyperintense (45-70 HU) extradural or subdural mass (Da Costa and Samii 2010).

The best imaging method employed in the diagnostics of degenerative changes of vertebral column, spinal neoplasms or disco-vertebral infections in dogs is MRI (Rossi et al. 2004; Hylands 2007; Da Costa and Samii 2010). It provides excellent information on the condition of the soft tissues, i.e. intervertebral discs, ligaments, meninges, epidural and subdural space, spinal cord and CE (Plate II, Fig. 4). The parasagittal and transverse (axial) reconstructions can even show the situation in vertebral foramina. That is why many of older techniques are gradually abandoned (Da Costa and Samii 2010).

However, it is important to emphasize that neither CT nor MRI are perfect. Results of these studies must always be correlated with clinical presentation (Suwankong et al. 2006). The same recommendation concerns the fact that clinical signs present in canine hip dysplasia (e.g. decreased activity, reluctance to rise, jump, run, climb stairs, lameness in the pelvic extremity, pain, stiffness, loss of thigh muscles) can mimic a situation seen in DLS and CES (Brisson 2010; Ginja et al. 2010; De Decker et al. 2014; Syrcle 2017). While the hip dysplasia is an orthopaedic disease characterized by joint laxity, grinding, pain and/or reduced range of motion in the coxo-femoral joint, but missing a neurological deficit, the fully developed CES reveals typical symptomatology of a L7-Cd5 spinal nerve roots lesion (Dewey 2008; Suwankong et al. 2008; Lorenz et al. 2011). The definite diagnosis may require X-rays, CT or MRI examination as well as electrodiagnostic studies which are able to exclude the nerve root entrapment (Szilasiová 2011; Shahmohammadi et al. 2016). 


\section{Therapy of cauda equina syndrome}

The majority of studies dealing with human CES emphasize that an early diagnosis and treatment before a sphincter dysfunction develops [i.e. sooner than the incipient cauda equina syndrome (CESI) progresses to the cauda equina syndrome with retention (CESR)], is crucial for a satisfactory outcome (Gleave and MacFarlane 2002; De Long et al. 2008). The same principle should be applied to animals (Fowler 1999). The treatment options for canine CES are conservative (medical) and surgical therapy (Linn et al. 2003; De Decker et al. 2014). The decision for one of these modalities depends on several variables, e.g. the age of the animal, intentions of the owner, the severity of clinical symptomatology, or the existence of serious comorbities (Palmer et al. 1991; Suw ankong et al. 2008).

The medical treatment may be effective in dogs with lumbosacral pain without deficit, especially in patients experiencing an initial episode of the disease (Dewey 2008). It consists of exercise restriction and administration of nonsteroidal anti-inflammatory drugs (NSAID). In the case of concomitant obesity, the diet is modified. The animal should be confined and allowed to walk only on leash for 4-6 weeks (Worth et al. 2009; Meij and Bergknut 2010). During this period, administration of NSAIDs is indicated (De Decker et al. 2014). However, NSAIDs can endanger the patient by gastrointestinal bleeding, development of gastroduodenal ulcers, arterial hypertension, and hepatic or renal complications. Diclofenac and ibuprofen have less serious side effects, and are therefore preferred currently (Viñuela-Fernández et al. 2007). Chemotherapy by cytostatic drugs is indicated in CES caused by chemosensitive malignant tumours (Pang and Argyle 2016).

In dogs with a light neurologic deficit, a combined administration of opioid analgesics or tramadol with corticosteroids can be used (e. g. methylprednisolone at 0.5 to $1.0 \mathrm{mg} / \mathrm{kg} /$ day for $2-4$ weeks, eventually $1.0 \mathrm{mg} / \mathrm{kg}$ methylprednisolone administered epidurally on days 1, 14, and 42; Brisson 2010). The side effects of corticosteroids are more frequent than those of NSAIDs, so their concomitant administration is strictly contraindicated (De Decker et al. 2014). The treatment of choice in dogs with CES caused by discospondylitis presenting by minimal neurologic deficit is the administration of antibiotics or antifungal drugs (following the identification of microorganisms) for 4-6 months (Rutges et al. 2016).

The efficacy of conservative management of DLS or CESI is variable (De Decker et al. 2014). Dogs with more pronounced symptomatology are more likely to benefit from surgical intervention (Linn et al. 2003). The timing of surgical decompression is not exactly defined, but if the critical compression of CE is exceeded, a neurological deficit will be an inevitable consequence. Thus, in dogs suffering from paresis/plegia and urinary retention, the relief of neural structures is urgent (De Long et al. 2008). Some neuroscientists believe that only this approach may prevent the development of intramedullary lesions and irreversible sequelae (Marsala et al. 1995; Orendacova et al. 2001). The type of surgical therapy depends on the aetiology. The principle is a thorough decompression of neural structures by a laminectomy which may be combined with a partial discectomy and nucleus pulpectomy (Palmer and Chambers 1991). The decompression can be combined with an internal fixation of the lumbosacral junction if L7/S1 instability is present (Johnson and Dunning 2005). The goal of surgery in animals with CES caused by traumatic events is the decompression of nerve roots, reposition of fragments, and stabilization of the vertebral column (Johnson and Dunning 2005). The fixation techniques require specific equipment and technical expertise. Therefore, they should be performed by an experienced veterinary orthopaedist (Palmer and Chambers 1991). A surgical intervention is indicated in CES caused by epidural or subdural haematoma, epidural abscess or subdural empyema and benign intraspinal neoplasms (Bagley and Gokaslan 2004; Kebaish and Awad 2004; Minish et al. 2015). 
The postoperative care is very important. It requires administration of analgesics (e.g. tramadol or opioids at 6-8 h intervals for 1-2 days) and strict reduction of physical activity for several days. Supportive care with intravenous fluid therapy should be provided until the animal begins to eat and drink (Palmer and Chambers 1991). If the patient is not able to urinate, it is necessary to empty urinary bladder with manual expression every 4-6 h (McMurray et al. 2006). In severely paraparetic or paraplegic animals, soft bedding and frequent repositioning help prevent the development of pressure sores. Some authors recommend three weeks of confinement to a small room or cage after the surgery, others permit the dog to walk slowly on a leash as soon as the actual condition of the animal allows it (Palmer and Chambers 1991). If necessary, additional support of pelvic limbs may be employed. Animals may require occasional medication for discomfort. A positive therapeutic effect of laser beam that facilitates tissue healing and regenerative processes may be utilized as well (Zielinska et al. 2017). A specific program of physiotherapy for several weeks should be outlined on an individual basis. It is aimed at regulating the dog's weight and improving its endurance, fitness and joint mobility (Nas et al. 2015).

\section{Prognosis}

The outcome in CES depends mainly on the aetiology and degree of the neurologic deficit, but several additional factors (e.g. the age of the dog, severity and chronicity of clinical signs, and assignation/further exploitation) also play their role in the prognosis (Linn et al. 2003; Suwankong et al. 2008). The majority of dogs with symptomatology limited to pain, reluctance to jump or tenderness when getting up will usually improve with rest, however, some of them may eventually need surgery. An overall improvement after surgery is reported in the range of 76-93\% (Suwankong et al. 2008). On the other hand, the recovery of neural functions in dogs with long-lasting symptomatology is generally slow and may never be complete in spite of thorough surgical release of neural structures, elimination of the underlying mechanical problem, proper after-treatment, and expert physiotherapy (Linn et al. 2003). For example, in a group of 156 surgically treated dogs with CES caused by DLS, walking on stairs, assuming a sitting position, and tail movement improved only in 57\%; stiffness, toe dragging, and tail tone in 50\%; and urinary incontinence in 38\% (Suwankong et al. 2008). That is why dogs older than 9 years with chronic pelvic limb paresis/plegia, muscle atrophy, urinary and/or faecal incontinence, and impotence due to CES are unlikely to return to previous activities or the breeding process (Linn et al. 2003; Webb et al. 2010).

The prognosis in spinal neoplasms is variable. Generally, it depends on the severity of the neurologic deficit, tumour type, location and duration of nerve root compression. Benign neoplasms (especially meningiomas and Schwannomas) and some malignant processes (e.g. plasma cell tumours) have a good prognosis with appropriate therapy (Bagley and Gokaslan 2004; Pang and Argyle 2016).

The prognosis in bacterial spinal infection depends on the response of microorganisms to antibiotic therapy. The outcome in CES caused by fungal discospondylitis or abscess is usually unfavourable (Velissaris et al. 2009).

The CES is a legitimate indication for euthanasia if permanent severe pain and/or neurological deficit cannot be relieved (Worth et al. 2009).

\section{Acknowledgements}

The collection and study of literature dealing with different aspects of CES as well as the preparation of the presented paper was supported by the VEGA grant No 1/0898/15 of the Ministry of Education, Science, Research and Sport of the Slovak Republic and the Slovak Academy of Sciences. The authors would like to express their gratitude to W. Bradford DeLong, MD for English language proofreading and to Silvia Ficova, DVM from SIBRA, Centre for Veterinary Medicine, Bratislava, Slovakia for providing the CT and MRI images. 


\section{References}

Bagley CA, Gokaslan ZL 2004: Cauda equina syndrome caused by primary and metastatic neoplasms. Neurosurg Focus 16: 11-18

Bergknut N, Smolders LA, Grinwis GCM, Hagman R, Lagerstedt A-S, Hazewinkel HAW, Tryfonidou MA, Meij BP 2013: Intervertebral disc degeneration in the dog. Part 1: Anatomy and physiology of the intervertebral disc and characteristics of intervertebral disc degeneration. Vet J 195: 282-291

Brisson A 2010: Intervertebral disc disease in dogs. Vet Clin North Am Small Anim Pract 40: 829-858

Da Costa RC, Samii VF 2010: Advanced imaging of the spine in small animals. Vet Clin N Am Small Anim Pract 40: $765-790$

De Decker S, Wawrzewski LA, Volk HA 2014: Clinical signs and outcome of dogs treated medically for degenerative lumbosacral stenosis: 98 cases (2004-2012). J Am Vet Med Assoc 245: 408-413

De Long WB, Polissar N, Neradilek B 2008: Timing of surgery in cauda equina syndrome with urinary retention: meta-analysis of observational studies. J Neurosurg Spine 8: 305-320

De Risio L, Thomas WB, Sharp NJ 2000: Degenerative lumbosacral stenosis. Vet Clin North Am Small Anim Pract 30: 111-132

Dewey CW 2008: A practical guide to canine and feline neurology. 2nd ed. Iowa State University Press, Ames, $706 \mathrm{p}$.

Fialová I, Paninárová M, Nečas A, Stehlík L, Proks P 2014: Prevalence of lumbosacral transitional vertebrae in dogs in the Czech Republic. Acta Vet Brno 83: 399-403

Fletcher TF 2013: Spinal cord and meninges. In: Evans HP, de Lahunta A (Eds): Miller's anatomy of the dog. $4^{\text {th }}$ edn. WB Saunders, St. Louis, pp. 589-610

Flückiger MA, Damur-Djuric N, Hässig M, Morgan JP, Steffen F 2006: A lumbosacral transitional vertebra in the dog predisposes to cauda equina syndrome. Vet Radiol Ultrasound 47: 39-44

Fowler CJ 1999: Neurological disorders of micturition and their treatment. Brain 122: 1213-1231

Ginja MM, Silvestre AM, Gonzalo-Orden JM, Ferreira AJ 2010: Diagnosis, genetic control and preventive management of canine hip dysplasia: a review. Vet J 184: 269-276

Gleave JR, MacFarlane R 2002: Cauda equina syndrome: what is the relationship between timing of surgery and outcome? Br J Neurosurg 16: 325-328

Hugo TB, Heading KL, Labuc RH 2014: Canine cerebrospinal fluid total nucleated cell counts and cytology associations with the prevalence of magnetic resonance imaging abnormalities. Vet Med Res Rep 4: 75-84

Hylands R 2007: Veterinary diagnostic imaging. Can Vet J 48: 429-432

Johnson AL, Dunning D, 2005: Atlas of orthopedic surgical procedures of the dog and cat. WB Saunders, St Louis, 247 p.

Kebaish KM, Awad JN 2004: Spinal epidural hematoma causing acute cauda equina syndrome. Neurosurg Focus 16: e1

Kobayashi S, Yoshizawa H, Yamada S 2004: Pathology of lumbar nerve root compression. Part 1: Intraradicular inflammatory changes induced by mechanical compression. J Orthop Res 22: 170-179

Lappalainen AK, Salomaa R, Junnila J, Snellman M, Laitinen-Vapaavuori O 2012: Alternative classification and screening protocol for transitional lumbosacral vertebra in German shepherd dogs. Acta Vet Scand 54: 27

Lee S, Chung CK, Hee S, Park SB 2013: Correlation between bone mineral density measurement by dual-energy $\mathrm{X}$-ray absorptiometry and Hounsfield units measurement by diagnostic CT in lumbar spine. J Korean Neurosurg Soc 54: 384-389

Linn LL, Bartels KE, Rochat MC, Payton ME, Moore GE 2003: Lumbosacral stenosis in 29 military working dogs: epidemiologic findings and outcome after surgical intervention (1990-1999). Vet Surg 32: 21-29

Lorenz MD, Coates JR, Kent M 2011: Handbook of Veterinary Neurology. WB Saunders, St. Louis, 545 p.

Marsala J, Sulla I, Jalc P, Orendacova J 1995: Multiple protracted cauda equina constrictions cause deep derangement in the lumbosacral spinal cord circuitry in the dog. Neurosci Let 193: 97-100

Mayhew PD, Kapatkin AS, Wortman JA, Vite CH 2002: Association of cauda equina compression on magnetic resonance images and clinical signs in dogs with degenerative lumbosacral stenosis. J Am Anim Hosp Assoc 38: $555-562$

Mazensky D, Flesarova S, Sulla I 2017: Arterial blood supply to the spinal cord in animal models of spinal cord injury. A review. Anat Rec 300: 2091-2106

McMurray G, Casey JH, Naylor AM 2006: Animal models in urological disease and sexual dysfunction. Br J Pharm 147: S52-S79

Meij B, Bergknut N 2010: Degenerative lumbosacral stenosis in dogs. Vet Clin N Am Small Anim Pract 40: 983-109

Minish KK, Chandrakant SK, Abay NM 2015: Spinal subdural haematoma. J Orthop Case Rep 5: 72-74

Musteata M, Nicolescu A, Solcan G, Deleanu C 2013: The 1H NMR profile of healthy dog cerebrospinal fluid. PLoS One 8: e81192 
Nas K, Yazmalar L, Sah V, Aydin A, Ones K 2015: Rehabilitation of spinal cord injuries. World J Orthop 6: 8-16

Nota SP, Braun Y, Ring D, Schwab JH 2015: Incidence of surgical infections after spine surgery: what is the impact of the definition of infection? Clin Orhop Relat Res 473: 1612-1619

Olmaker K, Holm S, Rosenquist AL, Rydevik B 1992: Experimental nerve root compression. Presentation of a model for acute, graded compression injury of the porcine cauda equina, with analyses of the normal nerve and vascular anatomy. Spine 16: 61-69

Orendacova J, Cizkova D, Kafka J, Lukacova N, Marsala M, Sulla I, Marsala J, Katsube N 2001: Cauda equina syndrome. Progr Neurobiol 64: 613-637

Otani K, Kikuchi S, Konno S, Olmaker K 2001: Blood flow measurement in experimental chronic cauda equina compression in dogs: changes in blood flow at various conditions. J Spin Disord 14: 343-346

Palmer RH, Chambers JN 1991: Canine lumbosacral disease. Part II. Definitive diagnosis, treatment and prognosis. Comp Cont Edu Pract Vet 13: 213-221

Pang LY, Argyle DJ 2016: Veterinary oncology: Biology, big data and precision medicine. Vet J 213: 38-45

Priester WA 1976: Canine intervertebral disc disease - occurrence by age, breed, and sex among 8117 cases. Theriogenology 6: 293-303

Rosc-Bereza, K, Kruszewski M, Ciach-Wysocka M, Boczarska-Jedynak JM 2013: Spinal epidural abscess: common symptoms of an emergency condition. Neuroradiol J 26: 464-468

Rossi F, Seiler G, Busato A, Wacker C, Lang J 2004: Magnetic resonance imaging of articular process joint geometry and intervertebral disk degeneration in the caudal lumbar spine (L5-S1) of dogs with clinical signs of cauda equina compression. Vet Radiol Ultrasound 45: 381-387

Rutges JP, Kempen DH, van Dijk M, Oner FC 2016: Outcome of conservative and surgical treatment of pyogenic spondylodyscitis: a systematic literature review. Eur Spine J 25: 983-999

Rydevik B 1993: Neurophysiology of cauda equina compression. Acta Orthop Scand 64: 52-55

Scharf G, Steffen F, Grünefelder F, Morgan JP, Flückiger M 2004: The lumbosacral junction in working German Shepherd Dogs - neurological and radiological evaluation. J Vet Med 51: 27-32

Shahmohammadi M, Khoushuod RJ, Zali A, Seddeghi AS, Kabir NM 2016: Examination of the predictive power of electromyography and urodynamic study in patients with cauda equina syndrome (horse tail syndrome). Acta Inform Med 24: 328-331

Suwankong N, Meij BP, Voorhout G, deBoer AH, Hazewinkel HAW 2008: Review and retrospective analysis of degenerative lumbosacral stenosis in 156 dogs treated by dorsal laminectomy. Vet Comp Orthop Traumatol 21: $285-293$

Syrcle J 2017: Hip dysplasia: clinical signs and physical examination findings. Vet Clin North Am Small Anim Pract 47: 769-775

Szilasiová J 2011: Electromyography - EMG. In: Šulla IJ (Ed.): Selected topics from neurosurgery. UPJŠ, Košice, pp. 85-96

Tan J, Shi J, Shi G, Liu Y, Liu X, Wang C et al 2013: Changes in compressed neurons from dogs with acute and severe cauda equina constrictions following intrathecal injection of brain-derived neurotrophic factor-conjugated polymer nanoparticles. Neural Reg Res 8: 233-243

Thongtrangan I, Le H, Park J, Kim DH 2004: Cauda equina syndrome in patients with low lumbar fractures. Neurosurg Focus 16: e6

Trebacz P, Galaty M 2016: Sciatic neuropathy caused by an intramuscular lipoma in dogs. Acta Vet Brno 85: 147-149

Velissaris D, Aretha D, Fligou F, Filos KS 2009: Spinal subdural Staphylococcus aureus abscess: case report and review of the literature. World J Emerg Surg 4:31

Viñuela-Fernández J, Jones E, Welsh E, Fleetwood-Walker SM 2007: Pain mechanisms and their implication for the management of pain in farm and companion animals. Vet J 174: 227-239

Webb AA, Ngan S, Fowler DJ 2010: Spinal cord injury II: prognostic indicators, standards of care, and clinical trials. Can Vet J 51: 598-604

Worth AJ, Thompson DJ, Hartman AC 2009: Degenerative lumbosacral stenosis in working dogs: current concepts and review. N Z Vet J 57: 319-330

Yoshizawa H, Kobayashi S, Kubota K 1989: Effects of compression on intraradicular blood flow in dogs. Spine 14: $1220-1225$

Zielinska P, Soroko M, Zwyrzykowska A, Kielbowicz Z 2017: The use of laser biostimulation in human and animal physiotherapy - a review. Acta Vet Brno 86: 91-96 
Plate I

Šulla I. et al.: Cauda equina syndrome ... pp. 321-330

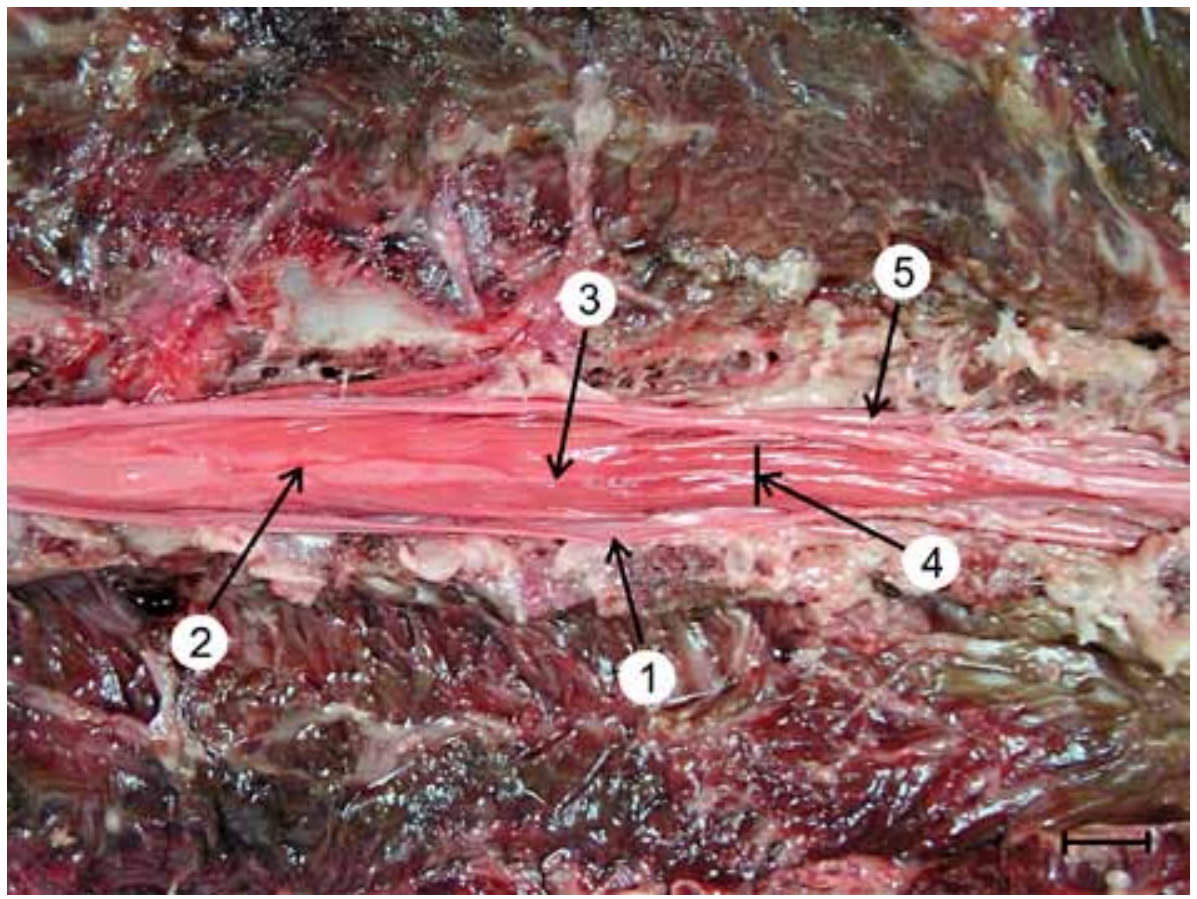

Fig. 1. An anatomical specimen of a German Shepherd dog depicting the caudal end of spinal cord. 1 - spinal dural sac; 2 - spinal cord; 3 - medullary cone; 4 - cauda equina nerve roots; 5 - spinal nerve. Scale bar $=5 \mathrm{~mm}$.

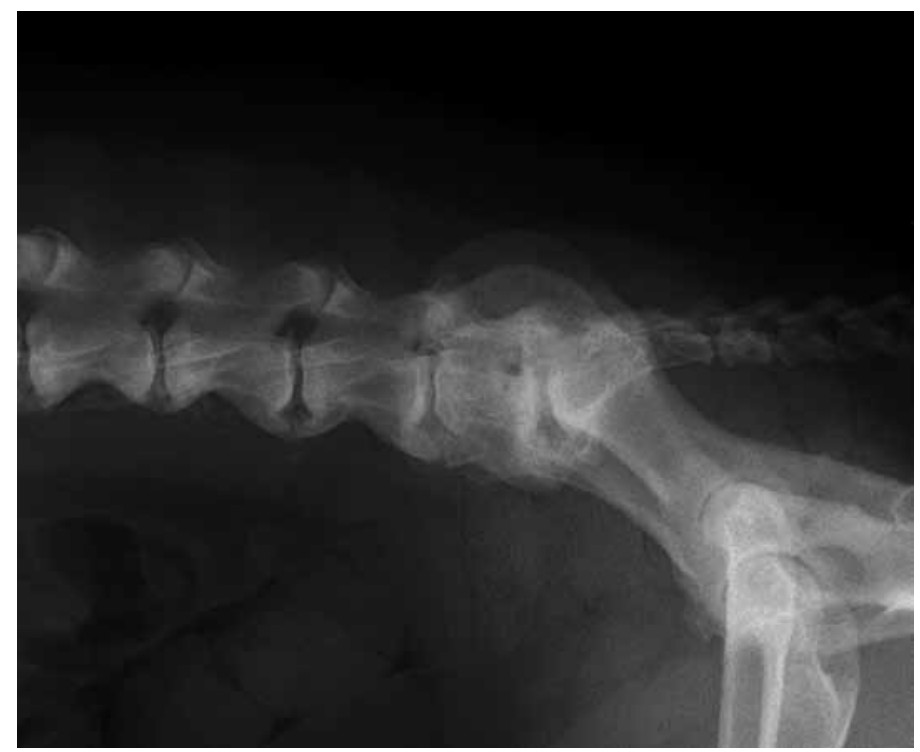

Fig. 2. An example of plain radiogram in LL view of a dog with degenerative changes of lumbosacral vertebral column. The intervertebral spaces are bridged by massive ventral osteophytes. Much more important are dorsal osteoproductive changes, which are smaller but cause spinal canal stenosis and potential compression of neural structures. 


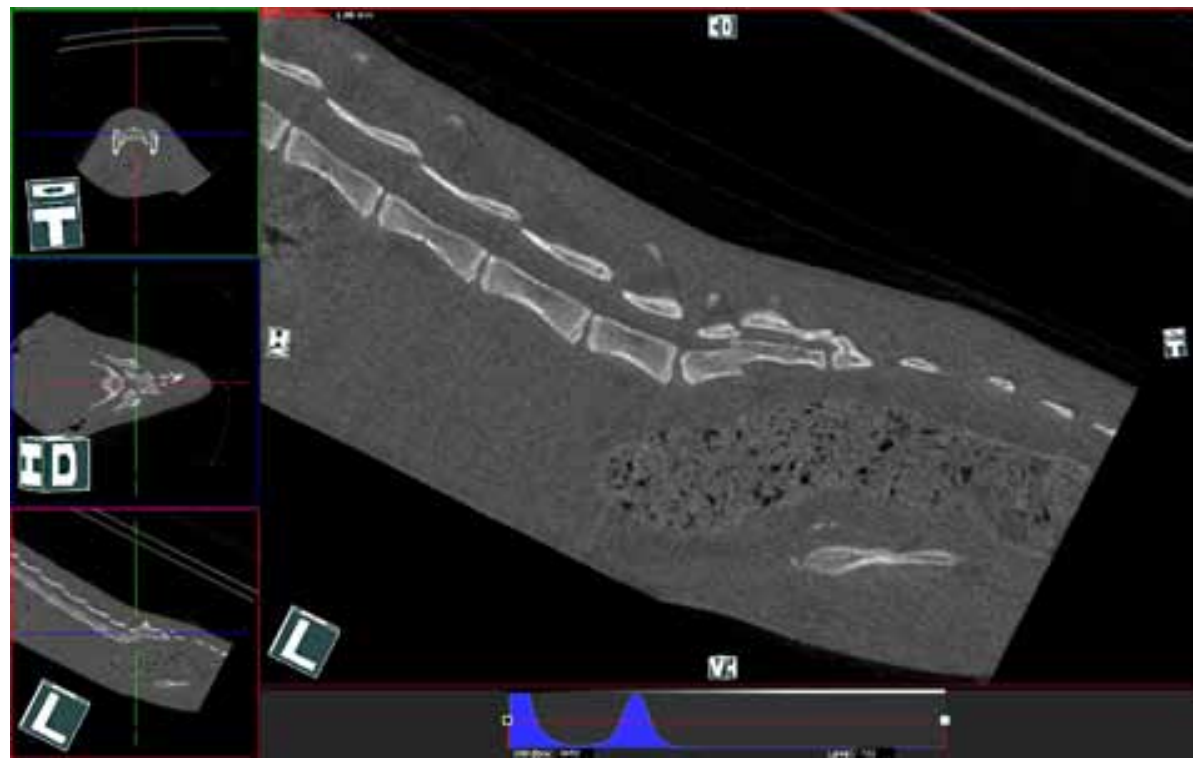

Fig. 3. Computed tomography examination of a dog with sacral bone fracture. The accumulation of faeces in the sigma and rectum (obstipation) is an indirect symptom of a traumatic cauda equina lesion and hypertonic anal sphincter. L - lateral view; T - transversal view; D - dorsal view.

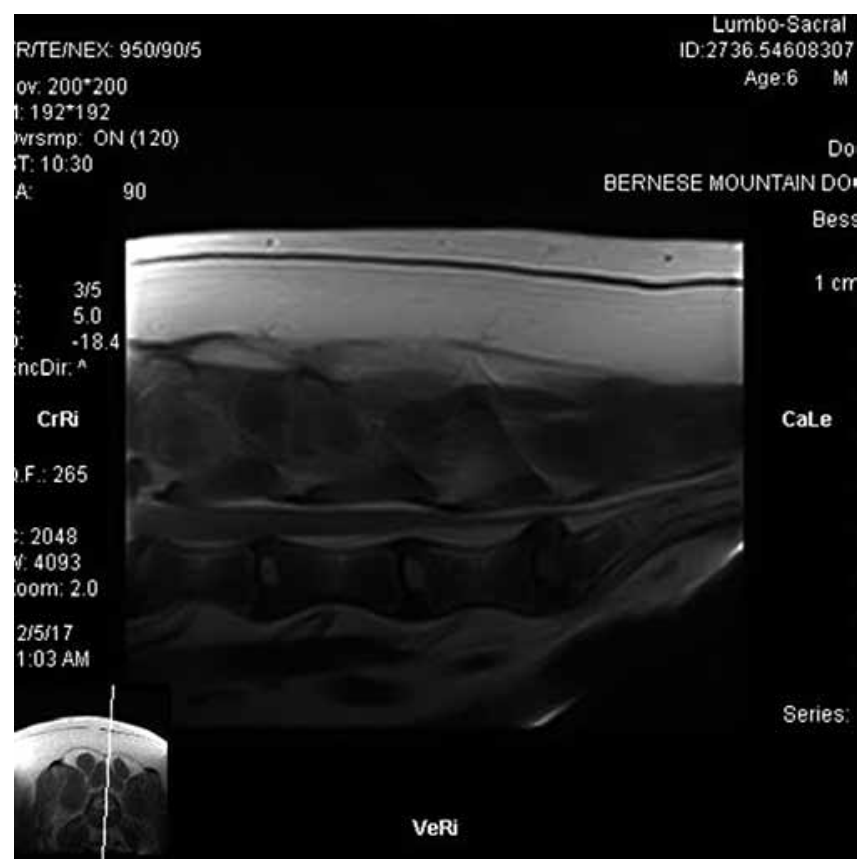

Fig. 4. Magnetic resonance imaging of a dog with Hansen type II L7/S1 intervertebral disc protrusion causing compression of cauda equina nerve roots; sagittal plane. 